\title{
Ultrasonic-Assisted Extraction and Evaluation of Biological Activities of Flavonoids from Flemingia philippinensis Merr et Rolfe
}

\author{
Hua Li, Qiyu Lu and Ke Bian* \\ Department of Food Science, Henan University of Technology, Zhengzhou, 450001, Henan, China
}

*For correspondence: Email: lixian78101@163.com; Tel/Fax: +86-371-67758022

Received: 18 March 2015

Revised accepted: 28 June 2015

\begin{abstract}
Purpose: To develop a simple and rapid method for extracting total flavonoids from the roots of Flemingia philippinensis and to investigate the antioxidant and anti-tumor activities of the extracts of the materials from various locations in China.

Methods: The total flavonoids in F. philippinensis were obtained by ultrasonic-assisted conventional solvent extraction method, and the extraction conditions were optimized by single factor and orthogonal test. 1,1-diphenyl-2-picrylhydrazyl (DPPH) radical scavenging and anti-tumor activities, using 3-(4,5dimethylthiazol-2-yl)-2,5-diphenyltetrazolium bromide (MTT) assay, of the extract were evaluated. The contents of flemiphilippinin A, auriculasin, 5,7,3', 4'-tetrahydroxy-6,8 -diprenylisoflavone and dorsmanins I were also determined.

Results: Optimal extraction conditions were as follows: extraction time, 40 min; methanol concentration, $85 \%$; and solvent to solid ratio, $40 \mathrm{~mL} / \mathrm{g}$; and number of extraction, once. Total flavonoid content varied greatly (3.7 - $14.35 \%)$ among the 19 samples collected from different origins in China. Flemiphilippin A, 5,7,3',4'- tetrahydroxy-6,8 -diprenylisoflavone, auriculasin and dorsmanins I showed varying DPPH radical scavenging activities with effective half maximal concentration $\left(E C_{50}\right)$ of 18.36, 23.59, 57.25 and $63.54 \mu \mathrm{g} / \mathrm{mL}$, respectively. Flemiphilippinin A (5 $\mathrm{g} / \mathrm{mL})$ also exhibited some level of antitumor activity against human hepatocellular carcinoma cell (BEL-7402), human lung epithelial (A-549) and human ileocecal adenocarcinoma cell (HCT-8) with inhibition of $91.13 \pm 1.6,91.22 \pm 3.23$, and $79.77 \pm 3.57 \%$, respectively.

Conclusion: Total flavonoids can be extracted efficiently from F. philippinensis by ultrasonic-assisted extraction method. Flemiphilippinin A has a potential for use in medicine as an antioxidant and antitumor drug.
\end{abstract}

Keywords: Flemingia philippinensis, Flemiphilippinin A, Ultrasonic-assisted extraction, Flavonoids, Antioxidant, Antitumor

Tropical Journal of Pharmaceutical Research is indexed by Science Citation Index (SciSearch), Scopus, International Pharmaceutical Abstract, Chemical Abstracts, Embase, Index Copernicus, EBSCO, African Index Medicus, JournalSeek, Journal Citation Reports/Science Edition, Directory of Open Access Journals (DOAJ), African Journal Online, Bioline International, Open-J-Gate and Pharmacy Abstracts

\section{INTRODUCTION}

Flemingia philippinensis Merr. et Rolfe, which belongs to the plant family Leguminosae, is mainly distributed in tropical areas of China. The traditional usage of the roots of $F$. philippinensis is for the treatment of rheumatism, arthropathy, leucorrhea, menalgia, menopausal syndrome, chronic nephritis, improving bone mineral density [1-3]. Modern pharmacological studies show that the extract from the roots of $F$. philippinensis exhibits anti-oxidative and anti-inflammatory activities due to its isoflavones [4]. The estrogenic and antiestrogenic bioactivities of the 
methanol extract were confirmed by the effects on the proliferation of MCF-7 cells and induction of $\beta$-galactosidase activity [5]. Flavonoids are the main constituents of $F$. philippinensis [3,6-12], and many of them have pharmacological activities. Total flavonoids can be used to evaluate the quality of the herb $[13,14]$.

To the best of our knowledge, there are no reports on total flavonoids content to evaluate the quality of the herb $F$. philippinensis and explore the antitumor activity. In this study, ultrasonicassisted extraction of total flavonoids and antioxidant and antitumor activity of the flavonoids isolated from $F$. philippinensis were carried out.

\section{EXPERIMENTAL}

\section{Chemicals and apparatus}

Nineteen samples (numbered 1 - 19) of $F$. philippinensis were collected from different locations in China, mainly in Guangxi Province, in the spring of 2009. They were identified by Professor Xiaojun Ma, Institute of Medicinal Plant Development, Chinese Academy of Medical Sciences and Peking Union Medical College. Voucher specimens (nos. FP001 - FP019) were deposited in the Herbarium of the Institute of Medicinal Plant Development, Chinese Academy of Medical Sciences, Peking Union Medical College. Standard genistein, flemiphilippinin $A$, auriculasin, 5,7,3',4'-tetrahydroxy-6,8 -diprenylisoflavone and dorsmanins I were purified by the first author to a level of $\geq 99 \%$, using a previously reported procedure [8-10].

All the cell lines were purchased from the Cell Culture Center of Institute of Basic Medical Science, Chinese Academy of Medical Sciences. 1,1-diphenyl- $\beta$-picrylhydrazyl (DPPH) and 3-(4,5dimethylthiazol-2-yl)-2,5-diphenyl-tetrazolium bromide were purchased from Sigma Chemical Co. while Medium RPMI-1640 was purchased from Gibico Co. All other reagents were of analytical grade.

\section{Total flavonoid extraction}

The roots were dried at $<60{ }^{\circ} \mathrm{C}$ in an oven, powered to 40 -mesh, weighed $(0.5027 \mathrm{~g})$ and placed in a $25 \mathrm{~mL}$ volumetric flask to which methanol was added to make up to volume. The flask was sealed, shaken well, and then extracted ultrasonically. The flavonoid content of the filtrate was determined spectrophotometrically at $258 \mathrm{~nm}$ (UV-1700 UV spectrophotometer, Shimadzu Corporation, Japan). The flavonoid content was calculated with reference to genistein standard. The flavonoid yield $(Y)$ was defined as the ratio of total flavonoid in the filtrate to total flavonoid in raw material.

\section{Extraction design}

The effect of sonication time (A), methanol concentration (B), solvent volume (C) and extraction times (D) on the yield of total flavonoids were investigated.

A three-level, four-factor, orthogonal design was employed, in which 9 experiments $\left(\mathrm{L}_{9} 3^{4}\right)$ were involved, and the flavonoid yield $(\mathrm{Y})$ was used as response in evaluating the extraction (Table 2). The factors and levels studied were determined based on the single factor experiments, such as A (20, 40 and $60 \mathrm{~min}), \mathrm{B}(70,85$ and $100 \%), \mathrm{C}$ $(10,20$ and $30 \mathrm{~mL})$ and $\mathrm{D}(1,2$ and 3 times).

\section{DPPH radical scavenging activity of extract}

DPPH radical scavenging activity was measured according to previous studies method [15]. Methanol solution of DPPH $2 \mathrm{~mL}$ of $0.2 \mathrm{mM}$ radicals was incubated with a series of different concentrations of the extract or flavonoids. The absorbance of the mixture was measured at 517 $\mathrm{nm}$ after $30 \mathrm{~min}$ of incubation at room temperature in the dark. Ascorbic acid (Vc) was used as the control and methanol as the blank.

The scavenging effect was calculated using equation 1.

Scavenging effect $\%=(1-A S / A B) \times 100 \ldots . .(1)$

where $A S$ and $A B$ stand for absorbance obtained for a sample (the extract, flavonoid or Vc) and the blank, respectively.

Table 1: Single factor experimental design

\begin{tabular}{lllll}
\hline & $\begin{array}{l}\text { Extraction time } \\
(\mathbf{A}, \mathbf{m i n})\end{array}$ & $\begin{array}{l}\text { Methanol } \\
\text { concentration }(\mathbf{B}, \mathbf{\%})\end{array}$ & $\begin{array}{l}\text { Solvent volume } \\
(\mathbf{C}, \mathbf{m L})\end{array}$ & $\begin{array}{l}\text { Extraction } \\
\text { times (D, times) }\end{array}$ \\
\hline 1 & $10,20,30,40,50,60$ & 70 & 20 & 1 \\
2 & 20 & $50,60,70,85,90,100$ & 20 & 1 \\
3 & 20 & 70 & $5,10,15,20,25,30$ & 1 \\
4 & 20 & 70 & 20 & $1,2,3$ \\
\hline
\end{tabular}


DPPH radical scavenging activity was plotted against sample concentration to obtain the $\mathrm{EC}_{50}$, which represents concentration of extract required to scavenge $50 \%$ of DPPH radicals.

\section{Antitumor test}

Human hepatoma cell line BEL-7402, human lung carcinoma cell line A-549 and human colorectal cancer cell line HCT-8 were cultured in RPMI-1640 medium with $10 \%$ new-born bovine serum (NBS), and incubated at $37{ }^{\circ} \mathrm{C}$ in a humidified atmosphere that contained $5 \% \mathrm{CO}_{2}$. For the experiment, cells were seeded into 96well plates at a density of $5 \times 10^{3}$ cells/well. After $24 \mathrm{~h}$, triplicate wells were treated with media and agents. Fluorouracil (5-FU) was used as positive control. After $72 \mathrm{~h}$ incubation at $37{ }^{\circ} \mathrm{C}$ in $5 \%$ $\mathrm{CO}_{2}$, the drug-containing medium was removed and replaced by $100 \mu \mathrm{L}$ fresh medium with 0.5 $\mathrm{mg} / \mathrm{mL}$ MTT solution. After $4 \mathrm{~h}$ incubation, the medium with MTT was removed and $200 \mu \mathrm{L}$ DMSO was added to each well. The plates were gently agitated until the color reaction was uniform and the OD570 was determined using a microplate reader (Wellscan MK3, Lab systems Dragon).

\section{Statistical analysis}

Each experimental value is expressed as the mean \pm standard deviation $(n=3)$. Statistical analysis was performed using Graphpad prism 5.0 and data analyzed using one way analysis of
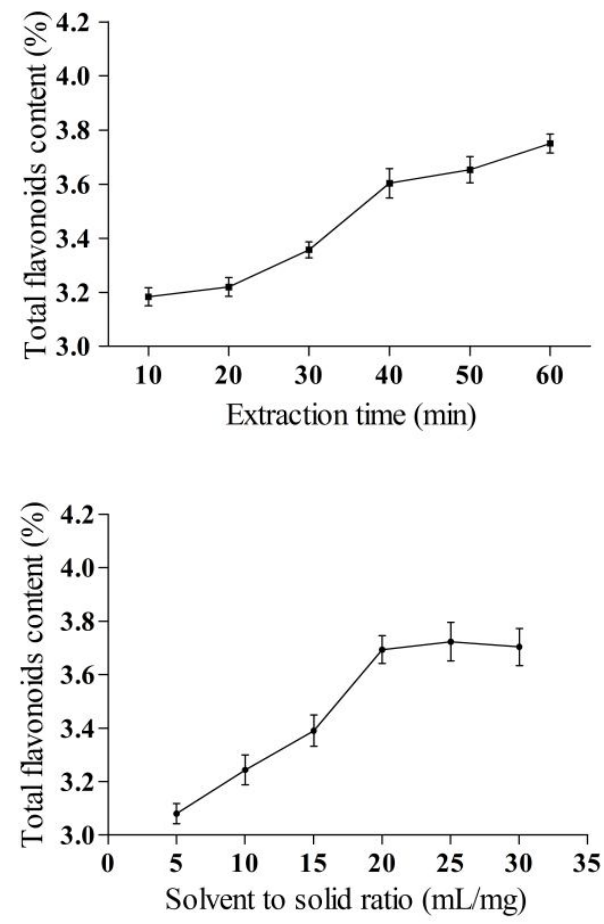

variance (ANOVA) for comparison between groups followed by Dunnett's multiple comparison test at a significant level of $p<0.05$.

\section{RESULTS}

\section{Optimization of extraction conditions}

As shown in Figure 1, the optimal conditions were as follows: sonication time, $40 \mathrm{~min}$; methanol concentration, $85 \%$; solvent volume, $20 \mathrm{~mL}$ and number of extraction, once for $0.5 \mathrm{~g}$ root powder.

The results of orthogonal design experiments were shown in Table 2. In order to keep consistent with single factor test, the extraction conditions were optimized as follows: sonication time, $40 \mathrm{~min}$; methanol concentration $85 \%$; solvent volume was $20 \mathrm{~mL}$ and number of extraction, once, at room temperature for $0.5 \mathrm{~g}$ of sample no. 4. The effect of the factors was in the rank order: $\mathrm{B}>\mathrm{A}>\mathrm{D}>\mathrm{C}$ in the ranges studied.

\section{Total flavonoid content}

The total flavonoid content in 19 samples were determined by calibration curve of genistein $(Y=$ $0.1726 X-0.0756$ with $\left.r^{2}=0.9996\right)$ and repeated three times for each under the optimal conditions. The results in Table 3 showed that the total flavonoid content were of great difference
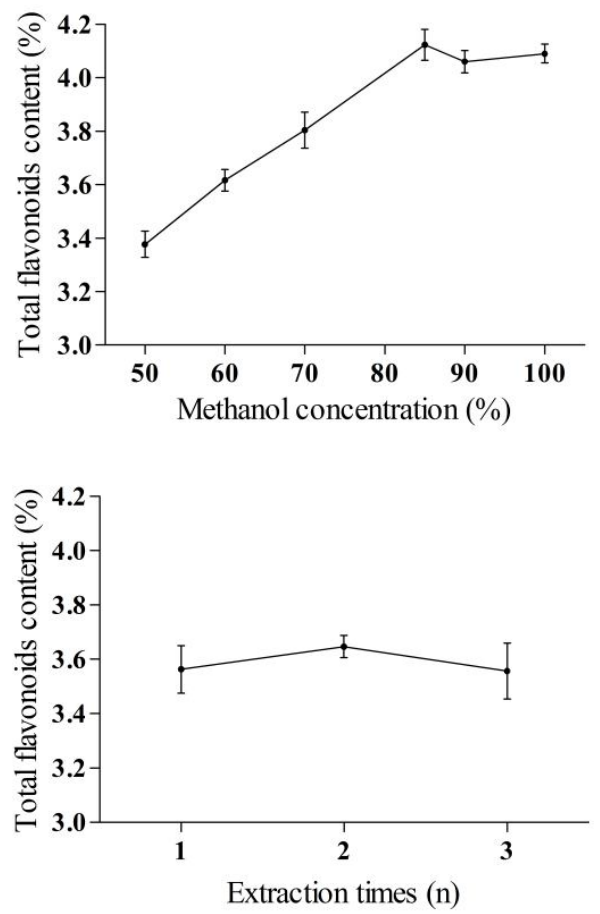

Figure 1: Effect of different levels of each factor on the yield of total flavonoids 
Table 2: Analysis and results of orthogonal design $\left(\mathrm{L}_{9} 3^{4}\right)$

\begin{tabular}{|c|c|c|c|c|c|}
\hline Variable & $\begin{array}{c}\text { Extraction } \\
\text { time }(A, \min )\end{array}$ & $\begin{array}{l}\text { Methanol } \\
\text { concentration (B, } \\
\%)\end{array}$ & $\begin{array}{c}\text { Solution } \\
\text { volume (C, } \\
\text { mL) }\end{array}$ & $\begin{array}{l}\text { Extraction } \\
\text { times(D, } \\
\text { times) }\end{array}$ & $\begin{array}{l}\text { Total flavonoids } \\
\text { content }\left(\mathrm{y}_{\mathrm{jk}}, \%\right)\end{array}$ \\
\hline 1 & 20 & 70 & 10 & 1 & 4.50 \\
\hline 2 & 40 & 85 & 20 & 1 & 4.75 \\
\hline 3 & 60 & 100 & 30 & 1 & 4.31 \\
\hline 4 & 40 & 70 & 30 & 2 & 4.38 \\
\hline 5 & 60 & 85 & 10 & 2 & 4.51 \\
\hline 6 & 20 & 100 & 20 & 2 & 4.25 \\
\hline 7 & 60 & 70 & 20 & 3 & 4.41 \\
\hline 8 & 20 & 85 & 30 & 3 & 4.38 \\
\hline 9 & 40 & 100 & 10 & 3 & 4.09 \\
\hline- & 4.38 & 4.43 & 4.37 & 4.52 & \\
\hline$y_{j 1}$ & & & & & \\
\hline $\bar{v}_{10}$ & 4.41 & 4.55 & 4.47 & 4.38 & \\
\hline 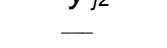 & 4.41 & 4.22 & 4.35 & 4.29 & \\
\hline $\begin{array}{l}y_{j 3} \\
\boldsymbol{R}\end{array}$ & 0.03 & 0.24 & 0.12 & 0.23 & \\
\hline $\begin{array}{c}\text { Optimized } \\
\text { level }\end{array}$ & $\mathrm{A} 2$ & B2 & $\mathrm{C} 2$ & $\mathrm{D} 1$ & \\
\hline
\end{tabular}

between 19 samples in which the lowest was $3.75 \%$ from Yunnan and the highest was 14.35 $\%$ from Daoxian Hunan. In addition, the content of total flavonoids in the xylem was different from that in phloem, the result showed that total flavonoids in xylem was much more than in phloem.

\section{Antioxidant activity}

The antioxidant activity of $85 \%$ methanol extract of $F$. philippinensis (specimen nos. 1 - 19) and flavonoid compounds isolated from Zhongshan guangxi sample is reported in Figure 2.

The extracts of 19 samples exhibited DPPH radical scavenging activities in the range $12.20 \pm$ $1.41 \%$ to $42.69 \pm 1.07 \%$ at concentration of 25 $\mu \mathrm{g}$ dry matter/mL, which suggested that the activities remarkably consisted with the content of their total flavonoids extracted under the optimal condition.

Four flavonoids, flemiphilippinin A, auriculasin, 5,7,3',4'-tetrahydroxy-6,8- diprenylisoflavone and dorsmanins I, exhibited DPPH radical scavenging activities in a concentration dependent manner, in the range of 5 to $50 \mathrm{mg} / \mathrm{mL}$ (Figure 2). Flemiphilippinin $A$ was found to have the highest DPPH scavenging activity (86.76\%), followed by auriculasin (79.38\%), 5,7,3',4'tetrahydroxy-6,8- diprenylisoflavone (51.67\%) and dorsmanins I (48.41\%), compared to a standard concentration of $50 \mathrm{mg} / \mathrm{mL}$ (Figure 2). The $E_{50}$ values $(\mu \mathrm{g} / \mathrm{mL})$ in descending order were flemiphilippinin A (18.36) > auriculasin
(23.59) > 5,7,3',4'-tetrahydroxy-6,8-diprenylisoflavone (57.25) > Dorsmanins (63.54), while the $\mathrm{EC}_{50}$ values of positive control Vc was 8.20 $\mu \mathrm{g} / \mathrm{mL}$.

\section{Antitumor activity}

The results for antitumor activities are presented in Table 3.

Flemiphilippinin A exhibited significant cytotoxic activity to three human cancer cell lines (HCT-8, A-549 and BEL-7402) in vitro, while three others showed no inhibitory activity against the three cancer cell lines. The antitumor activity of flemiphilippinin A against the three human cancer cell lines were quantitatively assessed further which was indicated in terms of $I_{50}(\mathrm{ug} / \mathrm{mL})$ values, which tested concentration were 0.005 , $0.05,0.5,5 \mathrm{ug} / \mathrm{mL}$. The results showed that flemiphilippinin $A$ exhibited a potent inhibitory effects on the indicated cell lines, especially for HCT-8, with an $\mathrm{IC}_{50}$ value of 1.17 , which higher than the positive control 5-FU with $I_{50}$ value 1.4. The $I_{50}$ values of flemiphilippinin $A$ inhibiting on A-549 and BEL-7402 were more than 5.0, which were higher than those of 5-FU, respectively, with $I_{50}$ value of 1.8 and 0.5 .

\section{DISCUSSION}

In the present study, total flavonoid was extracted and analyzed antioxidant activity using scavenging DPPH radical activity. The antioxidant and antitumor activities of the four 
Table 3: Total flavonoid content and DPPH radical scavenging activity of 19 samples from different locations in China

\begin{tabular}{|c|c|c|c|c|c|}
\hline No. & Sample & $\begin{array}{c}\text { Total flavonoids } \\
(\%)\end{array}$ & $\begin{array}{c}\text { Summary }^{a} \\
(p<0.05)\end{array}$ & $\begin{array}{c}\text { DPPH radical } \\
\text { scavenging activity } \\
(\%)\end{array}$ & $\begin{array}{l}\text { Summary }^{a} \\
(p<0.05)\end{array}$ \\
\hline FP001 & Lipu Guangxi & $5.29 \pm 0.15$ & ns & $14.29 \pm 2.70$ & ns \\
\hline FP002 & $\begin{array}{l}\text { Ningming } \\
\text { Guangxi }\end{array}$ & $10.57 \pm 0.35$ & $* * *$ & $26.17 \pm 0.68$ & $* * *$ \\
\hline FP003 & $\begin{array}{c}\text { Zhongshan } \\
\text { Guangxi }\end{array}$ & $11.54 \pm 0.08$ & $* * *$ & $32.50 \pm 1.39$ & $* * *$ \\
\hline FP004 & $\begin{array}{c}\text { Zhongshan } \\
\text { Guangxi }\end{array}$ & $5.00 \pm 0.56$ & -- & $16.78 \pm 1.67$ & -- \\
\hline FP005 & Laitu Guangxi & $9.38 \pm 0.06$ & $* * *$ & $24.65 \pm 2.38$ & $* * *$ \\
\hline FP006 & Jingxi Guangxi & $9.24 \pm 0.08$ & $* * *$ & $24.30 \pm 2.72$ & $* * *$ \\
\hline FP007 & Fuzhou Guangxi & $8.32 \pm 0.20$ & $* * *$ & $20.85 \pm 0.49$ & ns \\
\hline FP008 & Pingle Guangxi & $4.52 \pm 0.14$ & ns & $20.00 \pm 2.23$ & ns \\
\hline FP009 & $\begin{array}{l}\text { Gongcheng } \\
\text { Guangxi }\end{array}$ & $12.71 \pm 0.11$ & $* * *$ & $40.11 \pm 2.50$ & $* * *$ \\
\hline FP010 & $\begin{array}{l}\text { Longsheng } \\
\text { Guangxi }\end{array}$ & $9.29 \pm 0.04$ & $* * *$ & $25.52 \pm 1.15$ & $* * *$ \\
\hline FP011 & $\begin{array}{l}\text { Shangsi } \\
\text { Guangxi }\end{array}$ & $10.73 \pm 0.11$ & $* * *$ & $29.10 \pm 2.39$ & $* * *$ \\
\hline FP012 & $\begin{array}{l}\text { Rongshui } \\
\text { Guangxi }\end{array}$ & $5.37 \pm 0.50$ & ns & $18.03 \pm 2.19$ & ns \\
\hline FP013 & Jinxiu Guangxi & $4.50 \pm 0.13$ & ns & $15.60 \pm 1.42$ & ns \\
\hline FP014 & Tianlin Guangxi & $5.10 \pm 0.07$ & ns & $18.48 \pm 2.31$ & ns \\
\hline FP015 & Daoxian Hunan & $14.35 \pm 0.06$ & $* * *$ & $42.69 \pm 1.07$ & $* * *$ \\
\hline FP016 & $\begin{array}{l}\text { Chengdou } \\
\text { Sichuan }\end{array}$ & $4.48 \pm 0.09$ & ns & $18.90 \pm 2.55$ & ns \\
\hline FP017 & Sichuan & $4.81 \pm 0.13$ & ns & $21.27 \pm 1.29$ & * \\
\hline FP018 & Guangdong & $4.20 \pm 0.14$ & $* *$ & $16.53 \pm 0.59$ & ns \\
\hline FP019 & Yunnan & $3.74 \pm 0.36$ & $* * *$ & $12.20 \pm 1.41$ & * \\
\hline
\end{tabular}

${ }^{a}$ Statistical significance ( no. 4 sample acted as control, $p<0.05$ ); ${ }^{*}$ significant; ${ }^{* *}$ very significant; ${ }^{* *}$ highly significant; $n s=$ not significant; ${ }^{b} \mathrm{DPPH}$ radical scavenging activity was at concentration of $25 \mu \mathrm{g}$ dry matter $/ \mathrm{mL}$

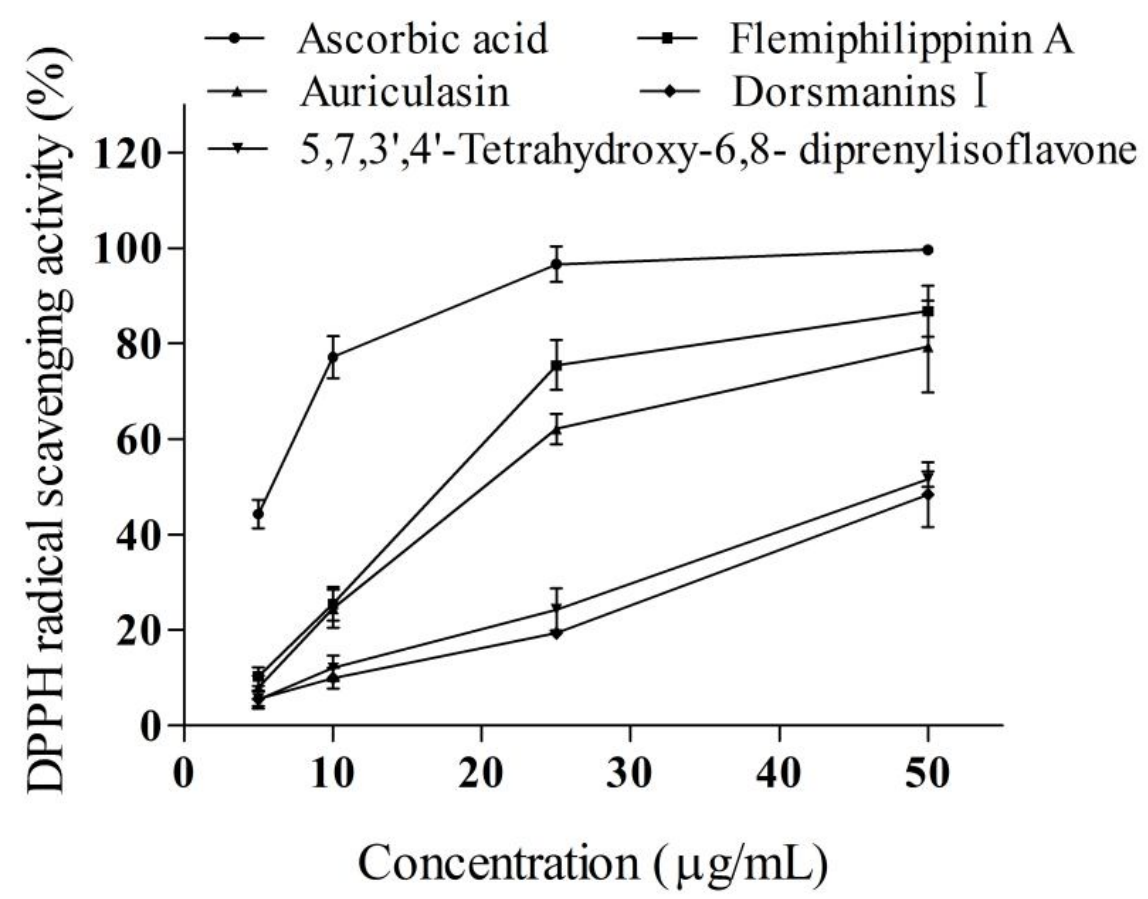

Figure 2: DPPH radical scavenging activity of flavonoids isolated from F. philippinensis 
Table 3: Anti-tumor activity of the extract and flavonoids from F. philippinensis (sample no. 4) used in preliminary screening

\begin{tabular}{|c|c|c|c|}
\hline \multirow[b]{2}{*}{ Sample * } & \multicolumn{3}{|c|}{ Inhibition rate (\%) } \\
\hline & BEL-7402 $^{\mathrm{a}}$ & $A-549^{D}$ & HCT-8 ${ }^{\mathrm{C}}$ \\
\hline Methanol extract & 39.67 & 44.84 & 47.91 \\
\hline Flemiphilippinin A & 91.13 & 91.22 & 79.77 \\
\hline Auriculasin & 64.98 & 3.33 & 47.35 \\
\hline $\begin{array}{l}5,7,3^{\prime}, 4^{\prime}-\text { Tetrahydroxy- } 6,8- \\
\text { diprenylisoflavone }\end{array}$ & 0.68 & 5.15 & 9.65 \\
\hline Dorsmanins I & 0.15 & 0.43 & 13.84 \\
\hline
\end{tabular}

${ }^{*}$ Anti-tumor activity determined at a concentration of $5 \mu \mathrm{g} / \mathrm{mL}$ for each samples; ${ }^{a} \mathrm{BEL}-7402=$ human hepatocellular carcinoma cell; ${ }^{b} A-549=$ human lung epithelial; ${ }^{c} H C T-8=$ human ileocecal adenocarcinoma cell

flavonoids isolated from F. philippinensis was also evaluated.

Ultrasonic-assisted extraction is a common and valid method to extract phytochemical compounds, including volatile constituents [16], flavonoid [17] and polysaccharides [18], etc. The orthogonal test design is a method that utilizes the orthogonal experiment tables to analyze experiment results scientifically. It provides a reasonable amount of information for testing the optimal combination from a fewer number of assays, therefore reducing more experiments associated with the analysis [19]. Under the optical extraction condition optimized with orthogonal design $\left(\mathrm{L}_{9} 3^{4}\right)$, total flavonoids concentration was in agreement with those evaluated by HPLC [20].

In the main peaks of the fingerprints, 14 peaks were identified by comparison of their retention times and UV absorption spectra with those of standard compounds which were separated from $F$. philippinensis. The identified peaks belonged to flavonoid. From the HPLC results, the most flavonoid compounds in this material were extracted under these extraction conditions. The total content of each flavonoid in the fingerprint was consistent with the result for total flavonoids. Therefore, using this method to extract and analyze total flavonoids in $F$. philippinensis is rational and feasible.

A preliminary study on antioxidant and antitumor activities of twelve flavonoids, isolated from $F$. philippinensis, suggested that there are only four flavonoids with antioxidant activities and one with antitumor activities. The results showed that the prenylated flavonoids (eg. flemiphilippinin A) had higher antioxidant activity than the prenylated ones (e.g., erythrinin B and genistin), this may be related to the hydroxyl number and threedimensional structure of the molecule, which can easily capture the free radicals caused by steric hindrance effect due to different prenyl groups. The underlying molecular phenomena of the radical scavenging activity of flavonoids can be explained by the ease of hydrogen atom abstraction and the ease of the termination of the flavonoid aroxyl radicals [21].

\section{CONCLUSION}

Among the materials tested, the plant material from Daoxian Hunan demonstrated the strongest radical scavenging, as well as the highest flavonoid contents. Flemiphilippinin A has high antioxidant and antitumor activities at low concentrations. The findings of this work also indicate that $F$. philippinensis is a potential new source of natural antioxidants and that flemiphilippinin $A$ can be developed as an antioxidant and antitumor agent with great commercial prospects in the pharmaceuticals industry.

\section{ACKNOWLEDGEMENT}

This work was supported by National Natural Science Foundation of China (no. NSFC31401548), Special Fund for Agroscientific Research in the Public Interest (no. 201303070) and Fundamental Research Funds for Special Projects of Henan University of Technology (no. 2014YWQQ04).

\section{REFERENCES}

1. Jiang Su New Medical College, Encyclopedia of Chinese Materia Medica, People's Publishing Press, Shanghai, 1977; p 212.

2. Li SG. China Flora, Science Press, Beijing, 1995; 41: 313-331.

3. Chen M, Luo SQ, Chen JH. Studies on the chemical constituents of Flemingia philippinensis. Acta Pharmacol Sin 1990; 26: 42- 48.

4. Pan BS, Kuo YY, Chen TY, Liu YC. Anti-oxidative and anti-inflammatory activities of two different species of a Chinese herb I-Tiao-Gung. Life Sci 2005; 77: 28302839.

Trop J Pharm Res, August 2015; 14(8): 1370 
5. Ahn EM, Nakamura N, Akao T, Nishihara T, Hattori M. Estrogenic and antiestrogenic activities of the roots of Moghania philippinensis and their constituents. Bio Pharm Bull 2004; 27: 548-553.

6. Ahn EM, Nakamura N, Akao T, Komatsu K, Qui MH, Hattori M. Prenylated flavonoids from Moghania philippinensis. Phytochem 2003; 64:1389-1394.

7. Chen M, Luo SQ, Chen JH. Two isoflavones fromFlemingia philippinensis. Phytochem 1991; 30: 3842-3844.

8. Li H, Yang MH, Miao JH, Ma XJ. Prenylated isoflavones from Flemingia philippinensis. Magn Reson Chem 2008; 46: 1203-1207.

9. Li H, Yang MH, Ma XJ. Flavonoids from roots of Flemingia philippinensis. Chin J Chin Mat Med 2009; 34: 724-726.

10. Li H, Yang MH, Si JY, Ma XJ. Chemical constituents from roots of Flemingia philippinensis. Chin Tradit Herb Drugs 2009; 40: 512-516.

11. Fu MQ, Deng D, Feng SX, Huang RM, Tian S, Qiu SX. Chemical Constituents from Roots of Flemingia philippinensis. Chin Herb Med 2012; 4: 8-11.

12. Wang Y. Curtis-Long MJ, Lee BW, Yuk HJ, Kim DW, Tan $X F$, Park $\mathrm{KH}$. Inhibition of tyrosinase activity by polyphenol compounds from Flemingia philippinensis roots. Bioorgan Med Chem 2014; 22:1115-1120.

13. Hosu A, Cristea VM, Cimpoiu C. Analysis of total phenolic, flavonoids, anthocyanins and tannins content in Romanian red wines: Prediction of antioxidant activities and classification of wines using artificial neural networks. Food Chem 2014; 150: 113118.
14. Wan PF, Sheng ZL, Han Q, Zhao YL, Cheng GD, Li YH. Enrichment and purification of total flavonoids from Flos Populi extracts with macroporous resins and evaluation of antioxidant activities in vitro. $J$ Chromatog B 2014; 945-946: 68-74.

15. Wang $H$, Gao XD, Zhou GC, Cai L, Yao WB. In vitro and in vivo antioxidant activity of aqueous extract from Choerospondias axillaris fruit. Food Chem 2008; 106: 888-895.

16. Jia $L H$, Liu $Y, L i Y Z$. Rapid determination of volatile constituents in safflower from Xinjiang and Henan by ultrasonic-assisted solvent extraction and GC-MS. J Pharm Anal 2011; 1: 213-218.

17. Liu BG, Ma YX, Liu Y, Yang Z, Zhang LP. UltrasonicAssisted Extraction and Antioxidant Activity of Flavonoids from Adinandra nitida Leaves. Trop J Pharm Res 2013; 12: 1045-1051.

18. Zheng Y, Lia Y, Wang WD. Optimization of ultrasonicassisted extraction and in vitro antioxidantactivities of polysaccharides from Trametes orientalis. Carbohydrate Polymers 2014; 111:315-323.

19. Liu SQ, Dai CF, Wang L, Lin SP, Li XD. Orthogonal test design for optimization of synthesis of MTX/LDHs hybrids by ion-exchange method. J Phys Chem Solids 2015; 79: 82-88.

20. Li H, Yang MH, Miao JH, Ma XJ. Simultaneous Chromatographic Fingerprinting and Quantitative Analysis of Flemingia philippinensis by LC-DAD. Chromatographia 2009; 70: 447-454.

21. Seyoum A, Asres K, El-Fiky FK. Structure-radical scavenging activity relationships of flavonoids. Phytochem 2006; 67: 2058-2070. 